\title{
Bone regeneration using injectable BMP-7 loaded chitosan microparticles in rat femoral defect
}

\author{
Venkata P. Mantripragada ${ }^{1}$, Ambalangodage C. Jayasuriya ${ }^{1,2^{*}}$
} \author{
of Toledo, Toledo, Ohio 43614-5807, USA. \\ *Author of Correspondence: Ambalangodage C. Jayasuriya, Ph.D. \\ University of Toledo \\ Department of Orthopaedic Surgery \\ 3065 Arlington Avenue \\ Dowling Hall \# 2447 \\ Toledo, OH 43614-5807 \\ USA
}

${ }^{1}$ Biomedical Engineering Program, ${ }^{2}$ Department of Orthopaedic Surgery, The University

Tel: 419-383-6557

Fax: 419-383-3526

E-mail: a.jayasuriya@utoledo.edu

Running Title: In vivo bone formation with BMP-7 loaded chitosan microparticles 


\begin{abstract}
Injectable chitosan microparticles were prepared using a simple coacervation method under physiologically friendly conditions by eliminating oil or toxic chemical, and employing low temperature and pressure for growth factor stability. Amount of $200 \mathrm{ng}$ of bone morphogenetic protein-7 (BMP-7) was incorporated in the chitosan microparticles by two methods: encapsulating and coating techniques. These microparticles were tested in vivo to determine the biological response in a rat femoral bone defect at 6 and 12 weeks. Four groups $(n=10)$ were tested which include two groups for BMP-7 incorporated microparticles (by two techniques), microparticles without BMP-7, and defect itself (negative control). Healthy bone formation was observed around the microparticles, which were only confined to the defect site and did not disperse. Histology indicated minor inflammatory response around the microparticles at 6 weeks, which reduced by 12 weeks. Micro-CT analysis of bone surface density and porosity was found to be significantly more $(\mathrm{p}<0.05)$ for microparticles containing groups, in comparison with controls, which suggests that the new bone formed in the presence of microparticles is more interconnected and porous. Collagen fibrils analysis conducted using multiphoton microscopy showed significant improvement in the formation of bundled collagen area (\%) in microparticles containing groups in comparison with controls, indicating higher cross-linking between the fibrils. Microparticles were biocompatibe and did not degrade in the 12 week implant period.
\end{abstract}

Keywords: chitosan microparticles; bone morphogenetic protein-7; in vivo; histology; microCT; multiphoton microscopy 


\section{Introduction}

Bone is a dynamic organ in the body that is constantly being resorbed and formed, through very meticulous processes. However, despite the excellent regenerative capacity of skeletal tissue, the biological process fails healing of large bone defects and fractures. Since several years, the gold standard treatment is the use of autografts, but its use is severely hampered by its short supply and the considerable donor site morbidity associated with the harvest. Risk of viral contamination and immunological response restrict the use of allografts. ${ }^{1-3}$

Therefore, research is still focused on developing ideal bone graft substitute material that promotes healing through osteogenesis, osteoconduction and osteoinduction, in combination or alone. The selection of an ideal bone graft depends on several factors such as defect size, graft size, shape and volume, tissue viability, biomechanical characteristics, graft handling, cost, ethical issues, biological characteristics and associated complications. ${ }^{4,5}$ Thus, optimizing scaffold design to satisfy all the requirements still remains a challenge. Apart from these characteristics, the biomaterial should finally degrade over time and be completely replaced by natural bone.

A number of biocompatible, biodegradable polymers - both natural and synthetic have been explored for repair and regeneration in the past decade. ${ }^{6-8}$ Chitosan is a natural polymer available in abundance. Chemically, it comprises of $\beta$ (1-4) linked D-glucosamine residues with N-acetylglucosamine side chains that makes it structurally similar to glucosaminoglycans (GAGs), which are usually the interacting molecules with osteoblasts, ${ }^{9-11}$ thus making it particularly attractive as a biomaterial for bone defects. Chitosan can also be degraded in vivo by lysozymes through hydrolysis of acetylated residues. It has also been found to provoke least foreign body response, 
leading to just normal granulation and neutrophils accumulation, which ultimately result in triggering local cell proliferation and integration of the scaffold with the host. ${ }^{12}$

Although chitosan has shown to be osteoconductive, it lacks osteoinductivity. Therefore, chitosan scaffolds with growth factors is being considered as a potential alternative. Our lab has been involved in developing chitosan scaffolds, with and without growth factors through various

techniques for application in bone tissue engineering. ${ }^{13-21}$ Therefore, the aim of the present investigation is to study the effects of chitosan microparticles with and without bone morphogenetic protein-7 (BMP-7) on bone healing in rats. Three groups of microparticles were used: chitosan microparticles without growth factors; chitosan microparticles with BMP-7 coated onto it; and BMP-7 encapsulated into it. Bone formation was analyzed at 6 and 12 weeks. The morphology of the newly formed bone was quantitatively analyzed using micro-computed tomography $(\mu-\mathrm{CT})$. It is known that collagen is an important part of bi-phasic bone composite material, which plays an important role in regulating its mechanical properties. Though there are many studies, which focus on mineral phase of bone, very few studies focus on the collagen content and arrangement to determine its role. Therefore, this study also analyses the collagen arrangement in the newly formed bone using a novel technique of multiphoton second harmonic generation microscopy. The biological response to the microparticles and growth factors and their degradation was observed by histology.

\section{Material and Methods}

\subsection{Materials}

Chitosan (85\% deacetylated, Molecular weight of 190 - $310 \mathrm{kDa}$ ), sodium tripolyphosphate (TPP), acetic acid, and phosphate buffered saline (PBS) were purchased from Sigma Chemicals, 
BMP-7 was purchased from Peprotech, 10\% formalin and decalcification solution, Cal-Ex 11 was obtained from Fisher Scientific.

\subsection{Methods}

\subsubsection{Fabrication of chitosan microparticles}

Microparticles were prepared by a coacervation technique. In this technique, $2 \%$ chitosan was prepared by dissolving in acetic acid (1\% v/v) at room temperature. The mixture was passed through a nylon mesh to remove insoluble substances. This mixture was then added drop wise to 50\% sodium tri-polyphosphate (TPP) solution kept on ice-bath. The microparticles were allowed

to cross-link overnight and then were air-dried. In order to prepare BMP-7 encapsulated microparticles, $200 \mathrm{ng}$ of BMP-7 was dissolved in PBS ( $\mathrm{pH}$ 7.4) and added to the chitosan solution before cross-linking. Each batch process resulted in a total of $7 \mathrm{mg}$ of microparticles. BMP-7 coated microparticles were prepared by adding the same amount of the growth factor to 7 mg of microparticles after they were cross-linked and dried through the process of absorption.

\subsubsection{Surgical Procedure}

Prior to the surgery, microparticles were packed in $1 \mathrm{ml}$ syringes along with very small amount of saline. These microparticles were sterilized using gamma radiation for 5 min at 1358.5 rads. Inbred male Lewis rats, 8 weeks old, weighing between 240-290 g (Harlan Laboratories, IN), were used as experimental animals. The protocol was approved by the University of Toledo Institutional Animal Care and Use Committee (IACUC, Approval no: 105818) and national guidelines for care and use of laboratory animals were followed.

Surgery was performed under general inhalation anesthesia containing isofluorane and oxygen. A lateral approach was used to expose the right femoral diaphysis. After the exposure of the mid 
shaft region of the femur, a $5 \mathrm{~mm} \times 1 \mathrm{~mm} \times 1 \mathrm{~mm}$ (length $\times$ width $\mathrm{x}$ height) hole was drilled through one of the cortex until the bone marrow was exposed. After injecting the material, the soft tissues and skin were closed layer-by-layer using absorbable suture.

Each group constituted 10 animals, thus for 4 groups (including a control) and two time points, a total of 80 animals were used in this study. At 6 and 12 weeks post-operation, rats were anesthetized using over dose of ketamine $(80 \mathrm{mg} / \mathrm{kg})$ and xylazine $(10 \mathrm{mg} / \mathrm{kg})$, followed by the cardiac perfusion technique to fix the tissue.

\subsubsection{Methods of evaluation of regenerated bone}

\subsubsection{Histological procedures}

The fixed tissue was decalcified before sectioning. Samples were embedded in liquid paraffin and cut into $5 \mu \mathrm{m}$ thick sections and fixed on microscopy slides for further analysis. The sections were stained with hematoxylin and eosin $(\mathrm{H} \& \mathrm{E})$.

\subsubsection{2 $\mu$-CT analysis}

Before decalcifying the bones, $\mu$-CT was performed. Samples were firmly positioned in the sample holder using low-density foam. In order to obtain the highest contrast between the specimen and the surrounding medium, air was used as the scanning medium for all the samples. The fixed scaffolds were scanned with a high-resolution $\mu$-CT scanner ( $\mu$ CT 35, Scanco Medical AG) at $70 \mathrm{kVp}$. Beam hardening of the X-ray was reduced by placing a beam-flattening filter in the X-ray path to narrow the energy spectrum. Signal-to-noise ratio (SNR) and scanning time had to be optimized to obtain good voxel information; therefore a tube current of $114 \mu \mathrm{A}$ was used with an integration time of $100 \mathrm{~ms}$ per projection. The tradeoff between voxel size and scan time was also carefully considered in order to analyze the newly formed bone in rat and an 
isotropic voxel size of $12 \mu \mathrm{m}$ was used for scanning all the bones. Region of interest (ROI) exceeding defect boundaries was used for scanning purposes (includes newly formed bone as well as old bone) and is approximately $20 \%$ of bone length. For each group $(n=10)$ samples were used.

The standard method of quantitatively describing bone architecture is the calculation of morphometric indices. Measurements such as bone volume fraction, specific bone surface, porosity of new bone, thickness of new bone fragments, spacing between newly formed bone fragments and number of new bone fragments were calculated using a preprogrammed algorithm to determine trabecular bone morphometry. But prior to computing the values of each of these outcome measures, a Gaussian filter (sigma $=0.8$, support=1.0) was applied for noise reduction. In order to determine all the above-mentioned parameters, contours were manually drawn carefully around the newly formed bone based on the visible threshold difference between the old and the new bone for all the groups. This threshold was carefully determined based on visual inspection of tomograms of regular cortical bone. Voxels above the threshold value of 289 and below 1000 were collected and the segmented bone was reconstructed for measurements.

\subsubsection{Multiphoton second harmonic generation}

Multiphoton second harmonic generation (SHG) imaging is based on the process of frequency doubling by which two near-infrared photons are converted into a single photon with exactly twice as much energy as the input photons. SHG signals arise due to interaction with anisotropic molecules, which in biological imaging, is most often present in collagen. We used Leica TCS SP5 laser scanning confocal microscopy (Leica Microsystems, Bannockburn, IL) equipped with a Ti-sapphire tunable multi-photon laser (Coherent, Santa Clara, CA). SHG for collagen was

optimally imaged using $860 \mathrm{~nm}$ excitation (MP laser) for maximum efficiency and emission 
collection was in the range of $425-435 \mathrm{~nm}$ with a peak emission generated at $430 \mathrm{~nm}$. H\&E stained slides were used for imaging. The images obtained were analyzed using ImageJ $1.48 \mathrm{j}$ version software $(\mathrm{NIH})$. During this analysis, collagen images were used to precisely match the collagen outline and converted to binary image for measurement. Three collagen attributes were acquired: total collagen area, collagen bundled area and collagen bundled area/total collagen area. Following an initial blind coded evaluation of 35 images, it was determined that an area of $3500 \mu \mathrm{m}^{2}$ was the minimum criterion for a collagen collection to be considered a collagen bundle. Accordingly, in each image, collagen bundle area with an area of at least $3500 \mu^{2}$ was summed to determine collagen bundled area of the entire image. The collagen bundle area was divided by the total collagen area to determine the relative amount of collagen bundling. For each group, $n=15$ images were processed.

\subsubsection{Statistical Analysis}

All statistical analyses were performed using SPSS software (IBM Corporation, Somers, NY, USA). A student T-test was applied for comparison of the groups at different implantation periods. Significant differences between the groups were determined using ANOVA's Tukey's Honest Significant Difference (HSD) test. The relationship between various parameters determined in this study was calculated by Pearson's regression coefficient. Results were considered significantly different if $\mathrm{p}<0.05$, unless otherwise mentioned.

\section{Results}

\subsection{Characterization of the microparticles}

Chitosan-TPP microparticles displayed a spherical and groovy surface morphology. The size of the microparticles obtained from a batch have an average diameter of $700 \mu \mathrm{m} .{ }^{13}$ The 
microparticles obtained by this method of cross-linking were found to have an internal less dense structure, which is advantageous in terms of growth factor delivery and degradation process. In order to incorporate BMP-7 into the microparticles, below room temperatures were employed to ensure structural and functional integrity of BMP-7. ${ }^{14}$

\subsection{Pre-clinical observations}

All the 80 rats operated exhibited good health and showed no complications throughout the study period. At the end of the postoperative time periods, i.e. 6 and 12 weeks, all the femurs were harvested and no visual signs of adverse tissue responses or inflammatory response were observed. In experimental groups, almost all the microparticles injected were found at the defect site, except in few, where the microparticles were in the adjacent muscle tissue due to the human error when injecting the microparticles (Fig. 1a,b).

\subsection{Histology observations}

\subsubsection{At 6 weeks}

In negative controls, where the defect was not filled with any material, a number of marrow filled cavities were observed around which new bone was being formed (Fig. 2a,b). Lining those cavities, we could observe active cuboidal osteoblasts forming new bone. In some of the spaces, multinucleated giant osteoclasts were also observed, suggesting active remodeling. Fibrous connective tissue/granulation tissue was also observed along the edges of the newly formed bone, which would later be mineralized to form woven bone. Lacunae were observed which had osteocytes. These cells can be seen very randomly arranged and in large population indicating the newly formed woven bone, which needs remodeling. 
In all the microparticle groups, the scaffolds appeared intact with minor surface degradation. Minor inflammatory response was confined to the region around the microparticles (Fig.2c,d). By carefully viewing all the images, we observed an increased inflammatory response in BMP-7 coated (Fig.2e,f) and encapsulated microparticles (Fig.2g,h) in comparison with microparticles without growth factor. Newly formed bone tissue was found in close proximity, around and in between microparticles. Active cuboidal osteoblasts were found on the uneven surfaces of the newly formed bone. In the marrow cavity, we could see osteoid being laid down by the differentiated osteoblasts. In BMP-7 encapsulated group, the marrow cavities appeared to be smaller in comparison with the other two microparticle groups and lot of woven bone growth was observed at the defect site. Blood vessels were formed in the new bone region. Fibrous tissue was found confined to the tissue-microparticle interface, which is consistent with postoperative changes. Loose granular-filamentous structure, containing collagen fibrils and fibroblasts is also found in-between microparticles. The morphological appearance of the newly formed bone was similar to the control and no necrotic tissue was observed indicating biocompatibility of the microparticles.

\subsubsection{At 12 weeks}

In controls, the number of marrow cavities and their size had dramatically reduced (Fig. 3a,b). We could still observe fibrous connective tissue/granulation tissue with fibroblasts, indicating active healing process. At the edges along the woven bone, we can observe active osteoblasts laying down bone (Fig. 3b). Well-developed blood vessels can be seen in the newly formed bone region. Osteoclasts were also observed in the small marrow cavities remodeling the bone.

In all the microparticle groups, the particles were still found to be intact, with lesser inflammatory response found around its perimeter (Fig. 3c,d) compared to that seen at 6 weeks. 
In BMP-7 coated and encapsulated groups, the inflammatory cells, were found penetrating the microparticle at 12 weeks (Fig. 3e,f,g,h). Similar to controls, we could observe active osteoblasts laying down bone on the newly formed uneven woven bone surface. Osteocytes were randomly arranged in the new woven bone indicating that remodeling is going on. The newly formed woven bone adjacent to the microparticle appeared tighter without interposition of fibrous tissue and seems to have increased at 12 weeks postoperatively. Blood vessel infiltration was also observed in newly formed bone region.

\section{$3.4 \mu-C T$ analysis}

\subsubsection{Bone volume fraction}

At 6 weeks, bone volume fraction, bone volume to total volume (BV/TV) in controls averaged $0.58 \pm 0.08$, for microparticles without growth factor it averaged $0.42 \pm 0.07$, for BMP-7 coated microparticles, the average is $0.38 \pm 0.10$ and for BMP-7 encapsulated microparticles, the average is $0.37 \pm 0.08$. Statistical analysis indicated a significant increase $(\mathrm{p}<0.001)$ in the BV/TV ratio in controls, in comparison with microparticles containing groups (with and without growth factors), also no significant difference is found in BMP-7 present and absent microparticles (Fig. 4a).

At 12 weeks, BV/TV ratio for controls is $0.58 \pm 0.05$ and showed no significant increase $(\mathrm{p}>0.05)$ from 6 weeks. For other groups-microparticles without growth factor, the ratio is $0.51 \pm 0.08$, BMP-7 coated microparticles, it is $0.48 \pm 0.06$ and for BMP-7 encapsulated microparticles, it is $0.46 \pm 0.08$ and these values indicate that BV/TV significantly increased in comparison with 6 weeks. There is also a significant increase $(\mathrm{p}<0.05)$ observed between the controls and the microparticles containing groups, but no significant difference was observed between 
microparticles and BMP-7 containing microparticles. The ratio of BV/TV was found to be 0.998 for positive controls (no defect) (Fig. 4b).

\subsubsection{Porosity of newly formed bone $\left(\mathrm{mm}^{-3}\right)$}

At 6 weeks, a significant difference $(\mathrm{p}<0.001)$ was observed in the porosity between the controls and microparticles containing groups (Fig. 4c). The controls averaged $85.88 \pm 20.73$, the microparticles average $204.92 \pm 69.25$, BMP-7 coated microparticles average to $203.31 \pm 79.78$ and BMP-7 encapsulated average to $194.85 \pm 65.58$. There was no significant difference observed between microparticles and BMP-7 containing microparticles.

At 12 weeks, there was a significant decrease $(\mathrm{p}<0.001)$ in porosity in comparison with its values at 6 weeks. Also, there was a significant difference $(\mathrm{p}<0.05)$ observed between the controls, which showed an average of $30.90 \pm 13.25$ and the microparticles containing groups-where microparticles indicated an average of $50.19 \pm 19.84$, BMP-7 coated microparticles have an average of 54.30 \pm 28.05 and BMP-7 encapsulated microparticles had an average of 54.43 \pm 24.11 . There was no significant difference observed between the BMP-7 containing microparticles and microparticles without any growth factors (Fig. 4d).

\subsubsection{Bone surface density $\left(\mathrm{mm}^{-1}\right)$}

At 6 weeks, bone surface density, bone surface to bone volume (BS/BV) ratio for controls averaged $15.81 \pm 2.51$ and this was significantly less $(\mathrm{p}<0.001)$ than the microparticles containing groups, where microparticles showed an average of $21.54 \pm 2.29$, BMP-7 coated averaged 23.47 \pm 5.33 and BMP-7 encapsulated averaged 24.46 \pm 8.12 . Statistical analysis indicated that there is no significant difference $(\mathrm{p}>0.05)$ between microparticles and BMP-7 containing microparticles (Fig. 4e). 
Even though at 12 weeks, there is no significant difference observed between the four groups, in comparison with 6 weeks there is a significant decrease $(\mathrm{p}<0.05)$ in the bone surface density (Fig. 4f), with controls showing an average of $12.15 \pm 1.69$, microparticles with an average of 11.79 \pm 1.97 , BMP-7 coated microparticles indicating about 12.75 \pm 1.64 and BMP-7 encapsulated microparticles showing an average BS/BV ratio of $12.21 \pm 1.62$.

\subsubsection{Newly formed bone fragment analysis}

Number of newly formed bone fragments $(1 / \mathrm{mm})$ at 6 weeks was averaged to be $7.52 \pm 0.51$ for controls, 6.41 \pm 2.43 in microparticle group, 6.53 \pm 2.90 in BMP-7 coated microparticles and 4.89 \pm 3.14 in BMP-7 encapsulated microparticles (Fig. 4g). But a decrease in bone fragments was observed at 12 weeks, controls showed an average of $5.71 \pm 0.49$, microparticles averaged $6.21 \pm 1.62$, BMP-7 coated microparticles averaged 5.01 \pm 2.32 and BMP-7 encapsulated microparticles had an average of $5.59 \pm 2.29$ trabecular number. At 6 and 12 weeks there was no significant difference observed in the trabecular number $\left(\mathrm{mm}^{-1}\right)$ between controls and microparticles containing groups, although there was a significant decrease $(\mathrm{p}<0.001)$ observed in the trabecular number from 6 weeks to 12 weeks in controls (Fig. 4h).

Newly formed bone fragment thickness $(\mathrm{mm})$ comparison indicated a significant difference $(p<0.001)$ between controls and microparticle groups at 6 weeks (Fig. 4i). Controls averaged $0.12 \pm 0.01$, whereas microparticles had an average of $0.10 \pm 0.01, \mathrm{BMP}-7$ coated microparticles had an average of $0.1 \pm 0.01$ and BMP-7 encapsulated microparticles had an average of $0.1 \pm 0.005$. There is no significant difference observed between microparticles and BMP-7 containing microparticles. At 12 weeks, a significant difference $(\mathrm{p}<0.05)$ was also observed between controls and microparticles containing groups. Controls had an average of $0.19 \pm 0.02$; while microparticles averaged to $0.17 \pm 0.02$, BMP-7 coated microparticles averaged to $0.16 \pm 0.02$ 
and BMP-7 encapsulated microparticles averaged to $0.16 \pm 0.02$. There was no significant difference observed between microparticles and BMP-7 containing microparticles (Fig. 4j).

Spacing between newly formed bone fragments $(\mathrm{mm})$ analysis at 6 weeks indicated that controls had an average spacing of $0.14 \pm 0.03$, while microparticles had an average of $0.25 \pm 0.18$, BMP-7 coated microparticles averaged to $0.28 \pm 0.26$ and BMP-7 encapsulated microparticles had an average spacing of $0.44 \pm 0.31$. There was a significant difference $(\mathrm{p}<0.001)$ observed between controls and BMP-7 encapsulated microparticles (Fig. 4k). At 12 weeks, the controls had an average spacing of $0.16 \pm 0.04$, microparticles averaged $0.25 \pm 0.2$, BMP-7 coated microparticles had an average of $0.43 \pm 0.35$ and BMP-7 coated microparticles had an average of $0.34 \pm 0.3$. There was a significant difference $(\mathrm{p}<0.05)$ observed between controls and BMP-7 coated and encapsulated microparticles (Fig. 41). There was no significant difference observed between BMP-7 containing microparticles and only the microparticles and also there was no significant increase in trabecular spacing observed between 6 and 12 weeks.

\subsubsection{Three Dimensional (3D) $\mu$-CT}

Fig. 5 and Fig. 6 are the three dimensional representation of the bone region scanned using micro-CT at 6 and 12 weeks respectively. These images indicate that due to the periosteal activity, a layer of bone has been formed at the periphery, but beneath that layer, there is still a lot of bone healing that needs to occur to bring back the original form. Fig. $5 \mathrm{c}$, and $6 \mathrm{e}$, we can clearly observe that microparticle is present at the defect site and new bone is being formed around it.

\subsection{Multiphoton second harmonic generation}


The bundled collagen fibrils constitute fibrils running parallel to each other. The newly formed region of bone constituted single fibrils, which have very small diameter, small bundled fibrils, with comparatively bigger diameter, and the thick bundled fibrils, with different dimensions, which may represent various stages of extracellular collagen organization. The fibrils organized into discrete bundled showed well-defined orientation. At 6 and 12 weeks, the images were analyzed using ImageJ and the regions in an image with an area greater than $3500 \mu \mathrm{m}^{2}$ (determined based on negative controls, data not mentioned here) were summed up to get the total bundled collagen area. Total collagen area was also determined in an image.

Fig. 7a,c,e,g are the bright field view of the new bone region analyzed and Fig. 7b,d,f,h are the corresponding multiphoton confocal microscopy images, respectively at 6 weeks post-surgery. The bundled collagen regions at the defect site were analyzed by ImageJ software using H\&E slides. At 6 weeks, average percent of bundled collagen is around $15.35 \pm 5.36$ for controls, $42.58 \pm 13.00$ for microparticles without growth factors, $41.63 \pm 10.55$ for BMP-7 coated microparticles and $49.91 \pm 18.09$ for BMP-7 encapsulated microparticles. It is observed that there is a significant difference in percentage of bundled collagen $(\mathrm{p}<0.005)$ between controls and microparticles containing groups (Fig. 9a). However, no significant difference was observed between microparticles containing BMP-7 and without BMP-7.

Similarly, at 12 weeks post-surgery, Fig. 8a,c,e,g are the bright field view of the new bone region analyzed and Fig. 8b,d,f,h are the corresponding multiphoton confocal microscopy images respectively. At 12 weeks, percent of bundled collagen averaged to $13.45 \pm 6.79$ for controls, for microparticles, the average is $38.09 \pm 16.13$, for BMP-7 coated microparticles, it averaged 49.06 \pm 17.15 and for BMP-7 encapsulated microparticles, the average is $32.50 \pm 15.06$. These results indicated a significant difference $(\mathrm{p}<0.05)$ between controls and microparticles containing 
groups (Fig. 9b). There was no significant difference observed in the percentage of bundled collagen between the BMP-7 present and absent groups. Also there was no significant decrease observed in the percent of bundled collagen between 6 and 12 weeks.

\section{Discussion}

The objective of this study is to examine the bone repair process at 6 and 12 weeks following application of chitosan-TPP and chitosan-TPP-BMP-7 scaffolds in femoral defect in skeletally matured rats.

A critical sized femoral defect model can serve as a robust test bed for tissue-engineered bone regeneration scaffold even in small animals such as rats. ${ }^{22}$ It was evident from the histology and $\mu$-CT data that the femoral defect did not heal completely in controls even after 12 weeks, suggesting that the defect model was appropriate for the study. $\mu$-CT and histology data suggests that bone formation was observed around and adhered to the microparticles. The rats used their injured leg to move and climb up the cage indicating that the microparticles have desirable mechanical properties to support the defect site.

Histology analysis was carried out to provide biologic insight to the various cellular events occurring during the complex healing process. Control defects in our study healed in a sequence which closely resembled natural fracture healing process, starting with clot formation, inflammatory response, followed by marrow derived stromal cell migration, vascularization, granulation tissue formation followed by bone formation by intramembranous and endochondral processes. Woven type of bone is formed during this process, which will be later remodeled. Even after 12 weeks post-surgery, we can observe soft cartilaginous callus formed around the 
newly formed bone in controls, which indicates that the bone healing process is still an ongoing process.

Studies have shown that following implantation of biomaterial, host reactions include bloodmaterial interaction, provisional matrix formation, acute inflammatory response, chronic inflammatory response, granulation tissue development, and foreign body reaction followed by fibrous capsule formation. ${ }^{23,24}$ The observations in our study were similar, wherein at 6 weeks, we could observe minor inflammatory response around the microparticles, and bone tissue growing in close proximity to the microparticles. Previous studies have shown that chitosan demonstrates chemotactic potential in vitro and in vivo. ${ }^{25-27}$ There are many studies, which showed minimal inflammatory response of chitosan, ${ }^{28-29}$ but there also exists many studies which show that chitosan could lead to high degree of inflammatory infiltrates ${ }^{30-31}$ and that reaction could last for long periods. ${ }^{32}$ But in our case, for chitosan-TPP and chitosan-TPP-BMP-7 microparticles fabricated by coacervation technique, we observed minor inflammatory response at week 6, which reduced by 12 weeks period. We observed that the granulation tissue is separated from the biomaterial by the cellular components of foreign body reaction where one or two layer of monocytes, macrophages and foreign body giant cells were present. These cells are mainly responsible for phagocytosis and degradation of the microparticles, which we started to observe by week 12. Fibrous tissue was observed around particles, which was similar to observations from other studies. ${ }^{33}$ The migration of cells is an important aspect of the stimulatory influence for tissue reconstruction. There is a noticeable difference in the inflammatory response observed between microparticles with and without BMP-7. In the microparticles with BMP-7, we could see the inflammatory cells making their way into the particles, which can lead to their early degradation. The possible explanation for this observation is that maybe due to the BMP-7 
released from the microparticles, cells migrate quicker to scaffold and induce scaffold degradation. $^{34}$

$\mu$-CT uses X-ray attenuation data acquired at multiple viewing angles to reconstruct a 3D representation of a specimen that characterizes the spatial distribution of material density. Therefore, quantitative analysis data for newly formed bone obtained by this method is more accurate. Bone volume fraction (BV/TV) results indicate that even though there is relatively less mineralized bone formed in microparticles group in comparison with controls, there is a significant increase $(\mathrm{p}<0.05)$ in the bone formation in the former groups at 12 weeks, which tells us that the microparticles support bone formation, but the degradation rate is slow and needs to be fastened. Bone volume fraction, is a ratio of bone volume and total volume. When individually these parameters were analyzed, we observed that in controls, there was a simultaneous increase in bone volume and tissue volume, which did not change the ratio, but in case of microparticles containing groups, there was twice an increase in bone volume in comparison with tissue volume between the two time points, therefore accounting for the significant difference. The fact that BV/TV ratio does not significantly change in controls confirms that the defect was critically sized for rats and is consistent with previous studies. ${ }^{35-37}$ No significant difference is observed between microparticles and BMP-7 containing microparticles, which can be explained by three reasons: (i) the quantity of BMP-7 incorporated was not sufficient to observe significant difference in bone growth. Even though our in vitro studies have demonstrated that BMP-7 being released from the microparticles is stable and bioactive, in vivo conditions are completely different, and results cannot be predicted definitely. ${ }^{13}$ Previous in vitro studies have also shown that femtomolar concentration of BMPs-3, -4 and -7 can initiate chemotaxis of monocytes. ${ }^{38,39}$ Nanogram level of BMP have shown 
mitogenic and osteogenic effects in cell culture, however macroscopic quantities of bone in vivo are induced only by milligram quantity of purified BMP or microgram range of rhBMP..$^{40,41,42}$ In addition, threshold levels also differ between species. A study reported that the use of $1.4 \mu \mathrm{g}$ BMP-2 in rat segmental defect in femur did not result in union, whereas a dose of $11 \mu \mathrm{g}$ was sufficient to complete union. In rabbits, $3.13 \mu \mathrm{g}$ of BMP-7 was insufficient to heal segmental ulna. These data suggest that the threshold of dose for in vivo bone induction is several magnitudes greater than that for cell response in vitro. (ii) The formation of fibrous capsule around the microparticles prevents the release of growth factor. Studies have shown that activated inflammatory cells (monocytes, macrophages) produce pro-fibrinogenic factors, which enhance fibrogenesis by fibroblasts. ${ }^{43}$ Therefore the macrophages adhered to the microparticle can secrete proteins that modulate fibrosis and in turn, fibrous capsule develops around the biomaterial (iii) the inflammatory cells (macrophages) that adhere and spread over the material surface release mediators of degradation such as reactive oxygen intermediates (ROIs, oxygen free radicals), degrading enzymes and acids into the zone between cell membrane and biomaterial surface. ${ }^{44}$ Biomaterial surface is therefore susceptible to high concentrations of these degradative agents ( $\mathrm{pH}$ as low as 4.0 ), which may have degraded the releasing protein. ${ }^{45}$ The new bone fragment thickness was averaged to be between 170-210 $\mu \mathrm{m}$ in controls and 140-190 $\mu \mathrm{m}$ for microparticles treated groups. These values were higher than those reported by other studies. ${ }^{46,47}$ TRI plate model was used to determine these values in both the cases and therefore discrepancy in the results can be due to difference in the calculation algorithm or the difference in the chosen threshold value. The number of new bone fragments was found to be about $5 \mathrm{~mm}^{-1}$ at the end of 12 weeks, which coincided with previously obtained results. ${ }^{47}$ 
Of the different parameters tested in this study, bone surface density and porosity density were found to be important parameters, which were significantly higher in microparticles containing groups in comparison with controls. Comparative analysis of $\mu \mathrm{CT}$ results show that $\mathrm{BS} / \mathrm{BV}$ and porosity of new bone show good Pearson's correlation coefficient value of $r=0.59(\mathrm{p}<0.001)$. Positive correlation factor indicates that more the surface area per bone volume, greater is the porosity. The presence of microparticles (with and without BMP-7) leads to increased BS/BV and porosity at 6 weeks, but with time, the porosity decreased, as observed at 12 weeks. By definition, connectivity density is a topologic measure counting the number of objects, the number of marrow cavity surrounded by bone and the number of connections that must be broken to split the structure into two parts. This supports the fact that the newly formed bone is more interconnected and porous in comparison with controls.

To further analyze the new bone, we determined the collagen fibrils formed in the new bone. This type of study is first of its kind to use confocal multiphoton second harmonic generation microscopy to determine the collagen fibril thickness and area in the newly formed bone and compare it between the scaffold-containing groups and controls. The capacity of the bone to resist mechanical forces and fractures depends on both the quantity of bone as well as on its quality. The quantity is in part evaluated by bone mineral density, which confers strength and stiffness to the tissue, ${ }^{48}$ whereas the quality in part is contributed by collagen phase, which confers ductility, playing a greater role in affecting the post yield properties of bone and overall toughness of the tissue. ${ }^{49,50}$ A study revealed that there is a 35\% decrease in strength, 30\% decrease in modulus and $50 \%$ decrease in toughness of collagen network with age, that results in failure of whole bone. ${ }^{50}$ Of the various structural and composition properties, collagen fibril diameter has been regarded as the most important factor related to biomechanical strength of the 
tissue. ${ }^{51}$ Our study revealed that microparticles containing femur defects showed significant increase in the percent of well-defined and organized bundled collagen area in the newly formed bone, in comparison with controls at both time points, which suggests the presence of microparticles, influences the mechanism of collagen fibrils formation. While it is evident that local microenvironment can influence fibril aggregation, it is also known that cells are responsible for molding tissue into specific configurations, by close spatial contact. Studies have shown that proteoglycans consisting of core protein and glycosaminoglycans (GAGs) have been implicated as regulators of collagen fibril I structure in different tissues. ${ }^{52}$ Though the information on precise role and mechanism by which the GAGs regulate collagen structure is limited, it can be explained that the presence of chitosan (containing structure similar to GAG) may be considered a cell-mediated modulary tool, which influence mesenchymal elements, as already documented for GAGs. These differences in fibril diameter have biomechanical implications. As we know, bone is mainly composed of type I collagen with type III and V

modulating the fibril diameter. ${ }^{53}$ Type I fibrils has larger diameter than type III collagen. ${ }^{54,55}$ Therefore, it may be postulated that new bone formed in the presence of microparticles (with and without BMP-7) has larger amount of type I collagen in comparison with controls.

\section{Conclusion}

Injectable chitosan microparticles combine favorable clinical handling properties with positive biological response in bone tissue. This study provides evidence that biocompatible, biodegradable and biomechanically stable chitosan scaffold can be developed by cross-linking with TPP at low temperatures, using minimum organic solvents, which accelerates better, and healthy bone formation compared to controls. This was illustrated by histology, which indicated 
healthy bone growth adhered to the periphery of the microparticles. This can be attributed to faster migration of cells towards BMP-7 containing microparticles. Bone surface density and connectivity density were found to be significantly high for microparticles containing groups, which suggest higher connectivity in the newly formed bone. This observation was also supported by confocal multiphoton second harmonic generation microscopy, where we observed a significant improvement in the formation of bundled collagen area (\%) in microparticle groups. This result indicates that the collagen fibril diameter is higher and hence the cross-link formation in collagen fibrils is higher. This result also suggests that perhaps, the newly formed bone is biomechanically superior. The influence of BMP-7 was noticed in histology, where we could observe microparticles degradation at 12 weeks. Apart from that there was no significant difference observed between microparticles with or without BMP-7, which suggests that the future studies would be oriented towards optimizing the concentration of BMP-7 being incorporated to observe a significant difference in vivo. The concentration should be such that it promotes bone formation but does not have any negative effects as observed in previous studies.

\section{Acknowledgements}

We would like to thank National Institute of Health (NIH) grant numbers R03DE019508 and R01DE023356 and National Science Foundation (NSF) grant number 1312465, for providing financial support to accomplish this work. We would like to thank Dr. Maged Hanna who assisted with the surgeries.

\section{References}

1. M. Aaboe, E.M. Pinholt, E. Hjorting-Hansen, Healing of experimental created defects: a review, Br J Oral Maxillofac Surg. 33 (1995) 12-18. 
2. J.S.Hermann, D.Buser, Guided bone regeneration for dental implants, Curr Opin Periodontol. 3 (1996) 168.

3. J.C. Douthitt, Guided tissue regeneration in surgical endodontics: improving the prognosis of periradicular surgery, Tex Dent J. 114 (1997) 8-12.

4. M.J. Yaszemski, R.G. Payne, W.C. Hayes, R. Langer, A.G. Mikos, Evolution of bone transplantation: molecular, cellular and tissue strategies to engineer human bone, Biomat.17 (1996) $175-85$.

5. A.J. Putnam, D.J. Mooney, Tissue engineering using synthetic extracellular matrices, Nature Med. 2 (1996) 824-26.

6. V.P. Mantripragada, B. Lecka-Czernik, N.A. Ebraheim, A.C. Jayasuriya. An overview of recent advances in designing orthopedic and craniofacial implants. J Biomed Mater Res A. 101 (2013) 3349-64.

7. M.B. Nair, H.K. Varma, K.V. Menon, et al., Reconstruction of goat femur segmental defects using triphasic ceramic-coated hydroxyapatite in combination with autologous cells and platelet-rich plasma. Acta Biomater 2009;5:1742-55.

8. A. El-Ghannam, L. Cunningham Jr, D. Pienkowski, et al., Bone engineering of the rabbit ulna, J Oral Maxillofac Surg, 65 (2007) 1495-1502.

9. R. Jayakumar, D. Menon, K. Manzoor, et al., Biomedical applications of chitin and chitosan based nanomaterials —A short review, Carbohydr Polym. 82 (2010) 227-232.

10. I.Y. Kim, S.J. Seo, H.S. Moon, et al., Chitosan and its derivatives for tissue engineering applications, Biotechnol. Adv. 26 (2008) 1-21. 
11. M. Peter, N.S.N. Ganesh, S.V. Nair, et al., Preparation and characterization of chitosangelatin/nanohydroxyapatite composite scaffolds for tissue engineering applications. Carbohydr. Polym. 80 (2010) 687-694.

12. J. Cui, J. Liang, Y. Wen, et al., In vitro and in vivo evaluation of chitosan/ $\beta$-glycerol phosphate composite membrane for guided bone regeneration, J Biomed Mater Res A. 102 (2014) 2911-2917.

13. V.P. Mantripragada, A.C. Jayasuriya, Injectable chitosan microparticles incorporating bone morphogenetic protein -7 for bone tissue regeneration: in vitro study, J Biomed Mater Res A. 102 (2014) 4276-4289.

14. V.P. Mantripragada, A.C. Jayasuriya, IGF-1 Release Kinetics and Osteoblast function of Chitosan Microparticles Fabricated Using Environmentally Benign Conditions, Mater Sci Eng C Mater Biol Appl 42 (2014) 506-516.

15. A.C. Jayasuriya, A. Bhat, Optimization of scaled-up chitosan microparticles for bone regeneration, Biomed Mater. 4 (2009) 055006.

16. A.C. Jayasuriya, A. Bhat, Mesenchymal stem cell function on hybrid organic/inorganic microparticles in vitro, J Tissue Eng Regen Med. 4 (2010) 340-348.

17. A.C. Jayasuriya, A. Bhat, Fabrication and Characterization of Novel Hybrid Organic/Inorganic Microparticles to apply in Bone Regeneration, J Biomed Mater Res A. 93 (2010) $1280-1288$.

18. A. Bhat, M. Dreifke, C. Gomez, et al., Evaluation of cross-linked chitosan microparticles for bone regeneration, J Tissue Eng Regen Med. 4 (2010) 532-542. 
19. A.C. Jayasuriya, A.Aryaei, A.H. Jayatissa, $\mathrm{ZnO}$ nanoparticles induced effects on nanomechanical behavior and cell viability of chitosan films, Mater Sci Eng C Mater Biol Appl. 33 (2013) 3688-3696.

20. A. Aryaei, A.H. Jayatissa, A.C. Jayasuriya, Mechanical and biological properties of chitosan-carbon nanotube nanocomposite films, J Biomed Mater Res A. 102 (2014) 27042712.

21. A. Aryaei, A.H. Jayatissa, A.C. Jayasuriya, Nano and Micro Mechanical Properties of CrossLinked Chitosan Samples, Journal of the Mechanical Behavior of Biomedical Materials, J Mech Behav Biomed Mater. 5 (2012) 82-89.

22. X. Chen, L.S. Kidder, W.D. Lew, Osteogenic protein-1 induced bone formation in an infected segmental defect in the rat femur, J Orthop Res. 20 (2002) 142-150.

23. D.T. Luttikhuizen, M.C. Harmsen, M.J. Van Luyn, Cellular and molecular dynamics in the foreign body reaction, Tissue Eng.12 (2006) 1955-1970.

24. C. Gretzer, L. Emanuelsson, E. Liljensten, et al., The inflammatory cell influx and cytokines changes during transition from acute inflammation to fibrous repair around implanted materials, J Biomater Sci Polym Ed. 17 (2006) 669-687.

25. Y. Okamoto, K. Shibazaki, S. Minami. Evaluation of chitin and chitosan on open would healing in dogs, J Vet Med Sci. 57 (1995) 851-854.

26. H. Ueno, H. Yamada, I. Tanaka. Accelerating effects of chitosan for healing at early phase of experimental open wound in dogs, Biomat. 20 (1999) 1407-1414.

27. Y. Usami, Y. Okamoto, S. Minami, Chitin and chitosan induce migration of bovine polymorphonuclear cells, J Vet Med Sci. 56 (1994) 761-762. 
28. C. Chatelet, O. Damour, A. Domard, Influence of the degree of acetylation on some biological properties of chitosan films, Biomat. 22 (2001) 261-268.

29. R. Muzzarelli, V. Baldassarre, F. Conti, et al., Biological activity of chitosan: ultrastructural study, Biomat. 9 (1988) 247-252.

30. Y. Hidaka, M. Ito, K. Mori, et al., Histopathological and immunohistochemical studies of membranes of deacetylated chitin derivatives implanted over rat calvaria, J Biomed Mater Res A. 46 (1999) 418-423.

31. R. Spin-Neto, R.M. de Freitas, C. Pavone, et al., Histological evaluation of chitosan-based biomaterials used for the correction of critical-size defects in rat's calvaria, J Biomed Mater Res A. 93 (2009) 107-114.

32. A.J. Asikainen, J. Hagstrom, T. Sorsa, et al., Soft tissue reaction to bioactive glass 13-93 combined with chitosan, J Biomed Mater Res A. 83 (2007) 530-537.

33. H. Chin, P. Quinten Ruhe, A.G.Mikos, In vivo bone and soft tissue response to injectable, biodegradable oligo(poly(ethylene glycol) fumarate) hydrogels, Biomat. 24 (2003) 32013211.

34. S. Shi, X. Cheng, J. Wang, et al., RhBMP-2 micropsheres-loaded chitosan/collagen scaffold enhances osseointegration: An experiment in dog. J Biomat Appl. 23 (2009) 331-348.

35. K. Ohura, C. Hamanishi, S. Tanaka, et al., Healing of segmental bone defects in rats induced by a b-TCP-MCPM cement combined with rhBMP-2, J Biomed Matr Res A. 44 (1999) 168-175.

36. C.A. Simmons, E. Alsberg, S. Hsiong, Dual growth factor delivery and controlled scaffold degradation enhance in vivo bone formation by transplanted bone marrow stromal cells. Bone. 35 (2004) 562-569. 
37. D.A. Oakes, C.C. Lee, J.R. Lieberman, An evaluation of human demineralized bone matrices in a rat femoral defect model. Clin Orthop Relat Res. 413 (2003) 281-290.

38. A.E. Postlethwaite, R. Raghow, G. Stricklin, L. Ballou, T.K. Sampath. Osteogenic protein-1, a bone morphogenic protein member of the TGF-beta superfamily, shares chemotactic but not fibrogenic properties with TGF-beta. Journal of Cellular Physiology, 1994161 562-570.

39. N.S. Cunningham, V. Paralkar, A.H. Reddi. Osteogenin and recombinant bone morphogenetic protein $2 \mathrm{~B}$ are chemotactic for human monocytes and stimulate transforming growth factor beta $1 \mathrm{mRNA}$ expression. Proceedings of the National Academy of Sciences of the USA 199289 11740-11744.

40. H. Mayer, A.M. Scutt, T. Ankenbauer. Subtle differences in the mitogenic effects of recombinant human bone morphogenetic proteins-2 to -7 on DNA synthesis in primary boneforming cells and identification of BMP-2/4 receptor. Calcified Tissue International 199658 $249-255$

41. B.D. Boyan, Z. Schwartz, L.D. Swain, A.G. Khare, J.D. Heckman, V. Ramirez, P. Peters, D. L. Carnes Jr. Initial effects of partially purified bone morphogenetic protein on the expression of glycosaminoglycan, collagen, and alkaline phosphatase in nonunion cell cultures Clinical Orthopaedics and Related Research 1992278 286-304.

42. M. R. Urist, K. Sato, A. G. Brownell, T. I. Malinin, A. Lietze, Y.K Huo, D. J. Prolo, S. Oklund, G. A. Finerman, R.J. DeLange. Human bone morphogenetic protein (hBMP). Proceedings of the Society for Experimental Biology and Medicine 1983173 194-199.

43. E. Song, N. Ouyang, M. Horbelt,et al., Influence of alternatively and classically activated macrophages on fibrogenic activities of human fibroblasts, Cell Immunol. 204 (2000) 1928. 
44. P. M. Henson. The immunologic release of constituents from neutrophil leukocytes. I. The role of antibody and complement on nonphagocytosable surfaces or phagocytosable particles, J Immunol.107 (1971) 1535-1546.

45. A.R. Amini, C.T. Laurensin, S. P. Nukavarapu. Bone Tissue Engineering: Recent Advances and Challenges. Crit Rev Biomed Eng 2012; 40(5): 363-408.

46. G. Lowett, G. Van der Perre, R. Van Audekercke, et al., Tiludronate improves the strength of vertebral bones in the rat after long-term administration, J Bone Miner Res. 12 (1997) S472.

47. T. Wronski, L. Dann, S. Horner, Time course of vertebral osteopenia in overiectomized rats, Bone. 10 (1989) 295-301.

48. J.D. Currey, The effect of porosity and mineral content on the Young's modulus of elasticity of compact bone, J Biomech. 21 (1988) 131-139.

49. P.Zioupos, Accumulation of in-vivo fatigue microdamage and its relation to biomechanical properties in ageing human cortical bone, J Microsc. 201 (2001) 270- 278.

50. X. Wang, R.A. Bank, J.M. TeKoppele, et al., The role of collagen in determining bone mechanical properties, J Orthop Res. 19 (2001) 1021-1026.

51. V. Ottani, M. Franchi, V. De Pasquale, et al., Collagen fibril arrangement and size distribution in monkey oral mucosa. J Anat. 192 (1998) 321-328.

52. D.R. Stamov, A. Müller, Y. Wegrowski, et al., Quantitative analysis of type I collagen fibril regulation by lumican and decorin using AFM. J Struct Biol.;183 (2013) 394-403.

53. S. Viguet-Carrin, P. Garnero, P.D. Delmas, The role of collagen in bone strength, Osteoporos Int.17 (2006) 319-336. 
54. T.F. Linenmayer, Collagen. In: Hay, E. (Ed.), Cell Biology of Extracellular Matrix, Vol. 2. Plenum Press, New York, London, pp. 1-44,1991.

55. M. Tzaphlidou, P. Berillis, Collagen fibril diameter in relation to bone site. A quantitative ultrastructural study, Micron, 36 (2005) 703-705. 


\section{Figure Captions}

Fig. 1. (a) The surgical procedure showing a 4-5 $\mathrm{mm}$ defect in diaphysial region of femur, which was implanted with chitosan-TPP microparticles (b) SEM image of chitosan-TPP microparticles.

Fig. 2. Microscopic images of transverse sections (H\&E) of rat femur at 6 weeks at 100x and 200x in (a,b) controls $(\mathrm{c}, \mathrm{d})$ microparticles group (e,f) BMP-7 coated microparticles group (g,h) BMP-7 encapsulated microparticles group respectively. CT, connective tissue; FB, fibroblasts; MP, microparticle; NB, new bone; OB, osteoblasts; OC, osteocytes; OCL, osteoclasts; OS, osteoid; IR, inflammatory response; BV, blood vessel (Scale bar represents $100 \mu \mathrm{m}$ ).

Fig. 3. Microscopic images of transverse sections (H\&E) of rat femur at 12 weeks at 100x and 200x in (a,b) controls (c,d) microparticles group (e,f) BMP-7 coated microparticles group (g,h) BMP-7 encapsulated microparticles group respectively. CT, connective tissue; FB, fibroblasts; MP, microparticle; NB, new bone; OB, osteoblasts; OC, osteocytes; OCL, osteoclasts; OS, osteoid; IR, inflammatory response; BV, blood vessel (Scale bar represents $100 \mu \mathrm{m}$ ).

Fig. 4. Box plot representation of a,b) Bone volume fraction (BV/TV); c,d) Porosity of new bone; e,f) Bone surface density (BS/BV); g,h) Number of new bone fragments; $i, j$ )

Fig. 5. Representative week 6 post-surgery micro-CT images of femurs for controls $(\mathrm{a}, \mathrm{b})$ and treated groups- microparticles (c,d); BMP-7 coated microparticles (e,f); BMP-7 encapsulated microparticles $(\mathrm{g}, \mathrm{h})$. Scale bar $=1 \mathrm{~mm}$

Fig. 6. Representative week 12 post-surgery micro-CT images of femurs for controls (a,b) and treated groups- microparticles (c,d); BMP-7 coated microparticles (e,f); BMP-7 encapsulated microparticles $(\mathrm{g}, \mathrm{h})$. Scale bar $=1 \mathrm{~mm}$. 
Fig.7. Representative histological section (H\&E) as observed by multiphoton confocal microscopy under bright field view and the corresponding view of collagen fibers in the newly formed bone region (ROI) at 6 weeks for $(a, b)$ controls $(c, d)$ microparticles group (e,f) BMP-7 coated microparticles group (g,h) BMP-7 encapsulated microparticles group respectively. (Scale bar represents $250 \mu \mathrm{m})$.

Fig.8. Representative histological section (H\&E) as observed by multiphoton confocal microscopy under bright field view and the corresponding view of collagen fibers in the newly formed bone region (ROI) at 12 weeks for $(a, b)$ controls $(c, d)$ microparticles group (e,f) BMP-7 coated microparticles group (g,h) BMP-7 encapsulated microparticles group respectively. (Scale bar represents $250 \mu \mathrm{m})$.

Fig. 9. Box plot representation of bundled collagen formed in the new bone region (ROI) obtained by analyzing the confocal multiphoton microscopy images $(n=15)$ using Image J software at a) 6 weeks b) 12 weeks. 


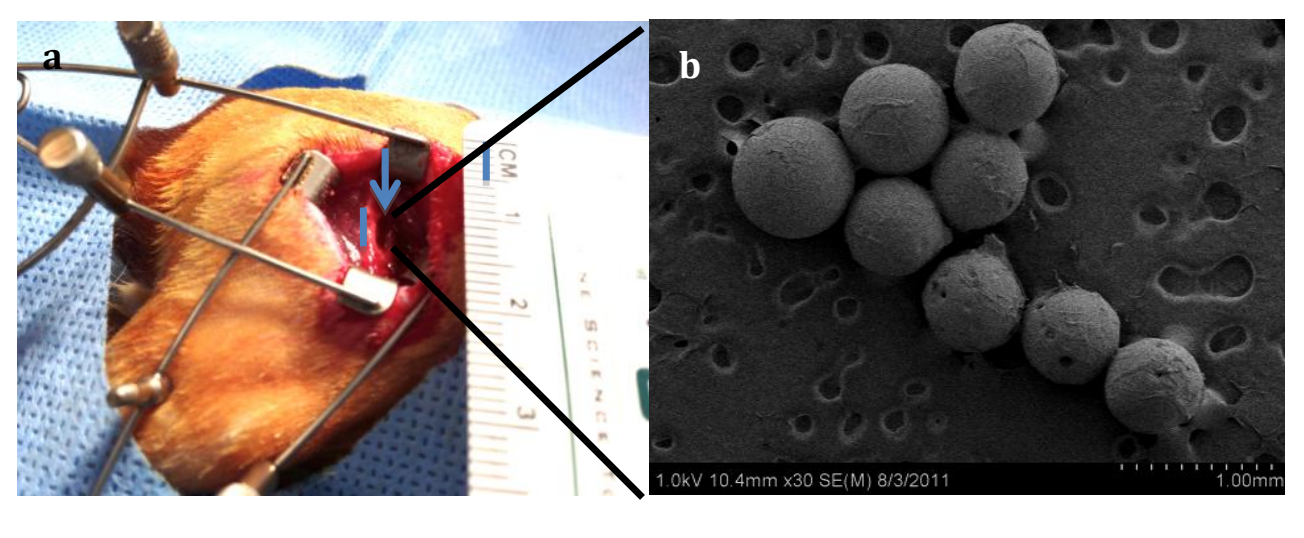

Fig. 1

Figure

Fig. 1

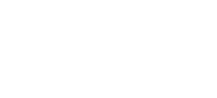

.

Fure

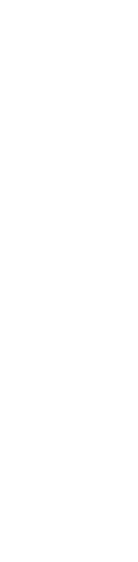

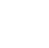

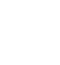


6 weeks

Controls

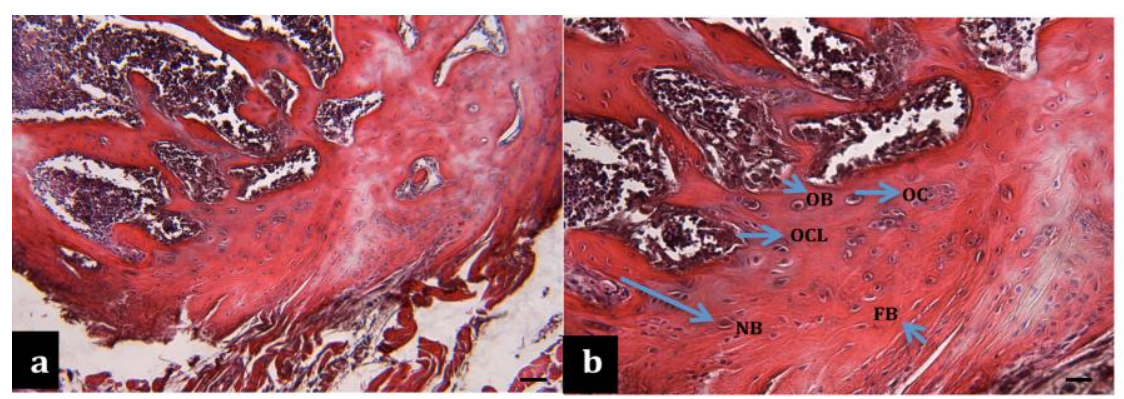

Microparticles

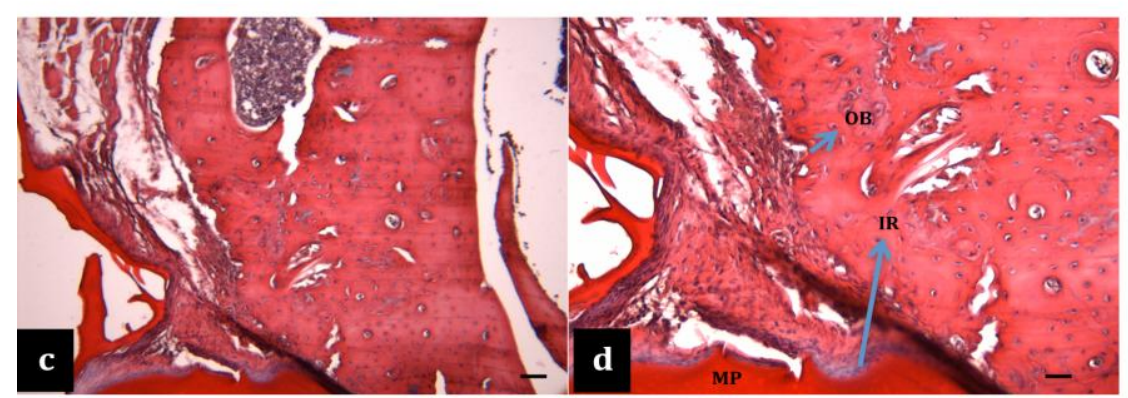

BMP-7 coated microparticles

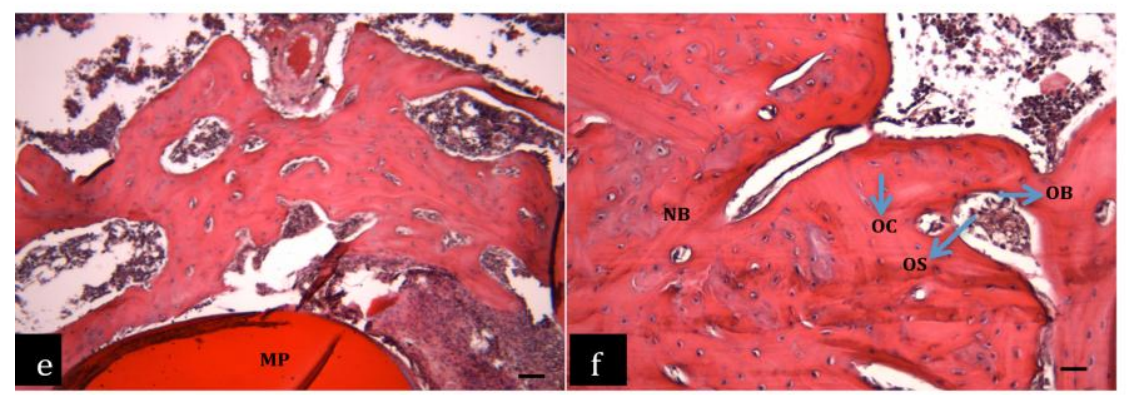

BMP-7

encapsulated microparticles

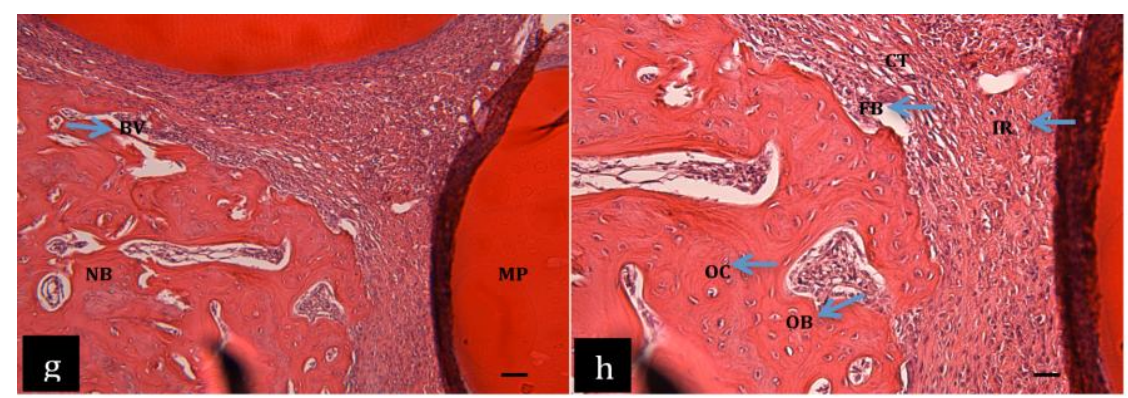

Fig. 2. 
12 weeks

Controls

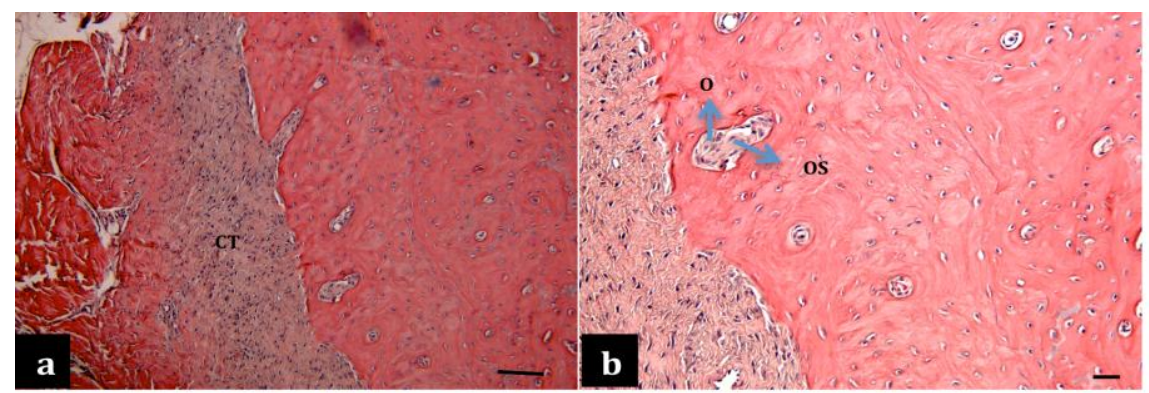

Microparticles

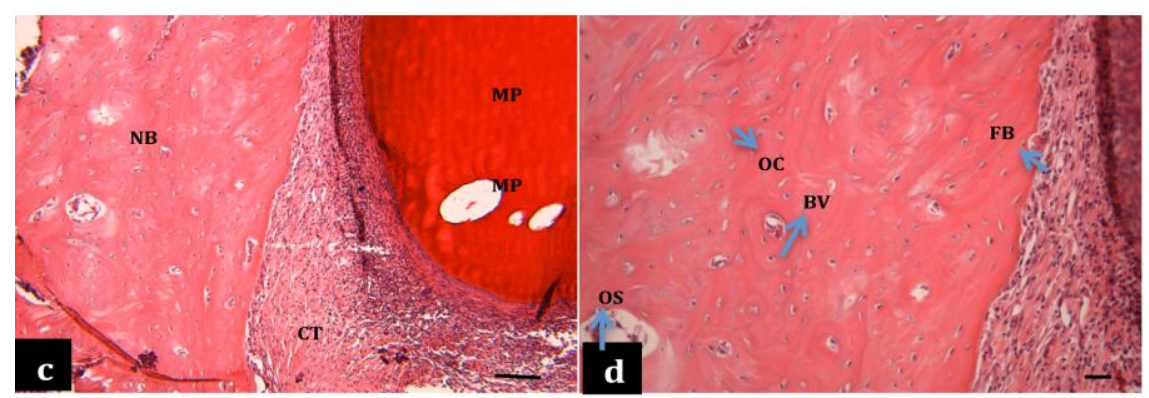

BMP-7 coated microparticles

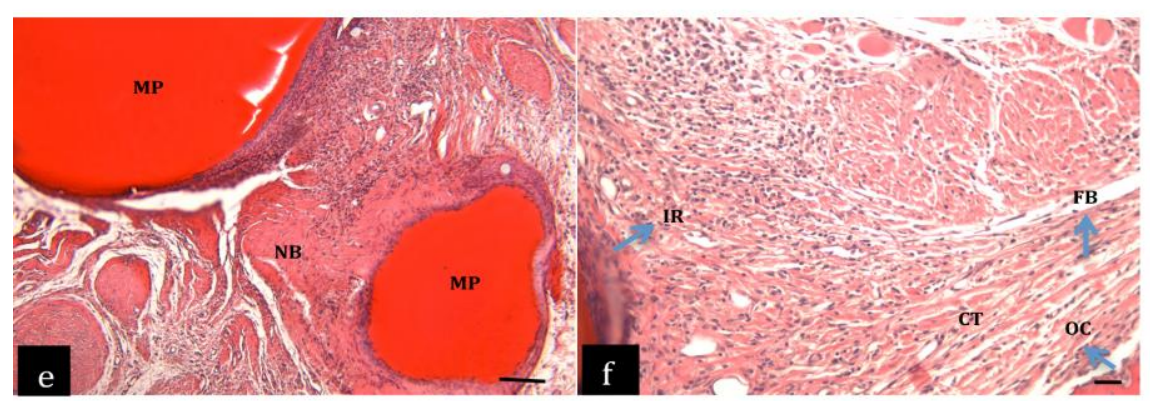

BMP-7
encapsulated
microparticles

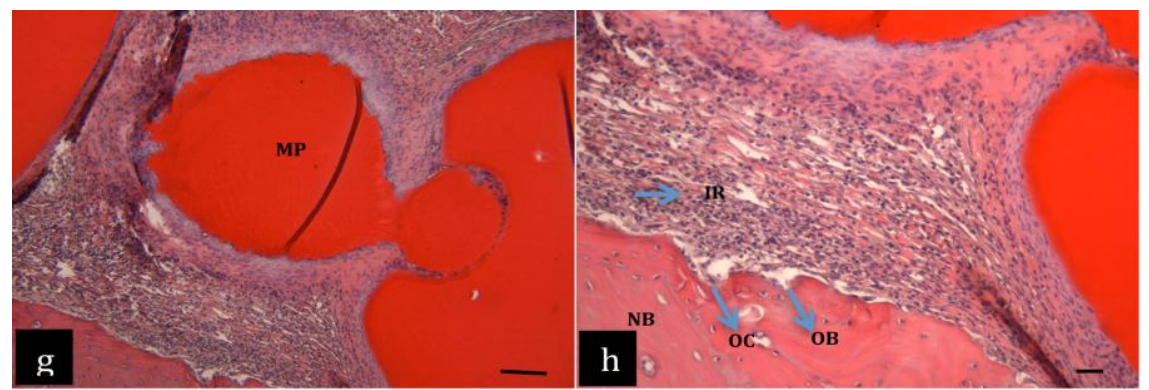

Fig. 3. 


\section{6 weeks}
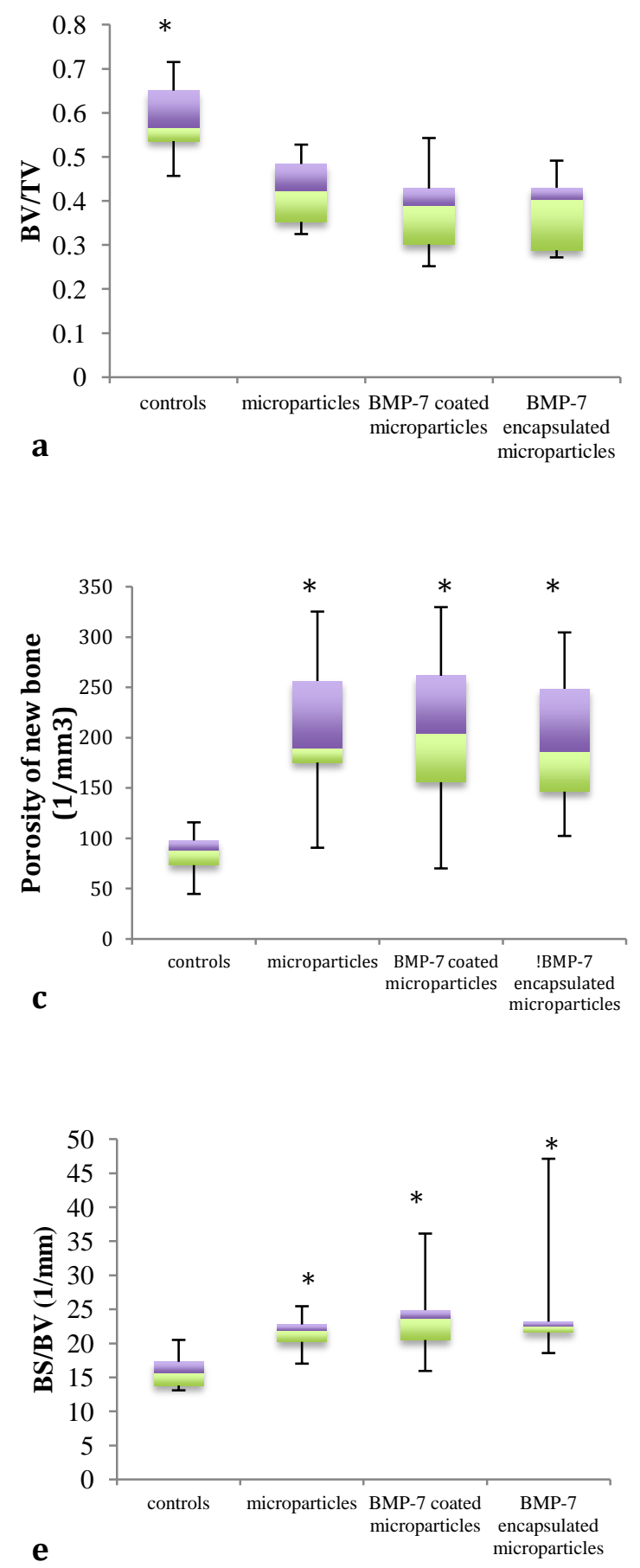

\section{2 weeks}
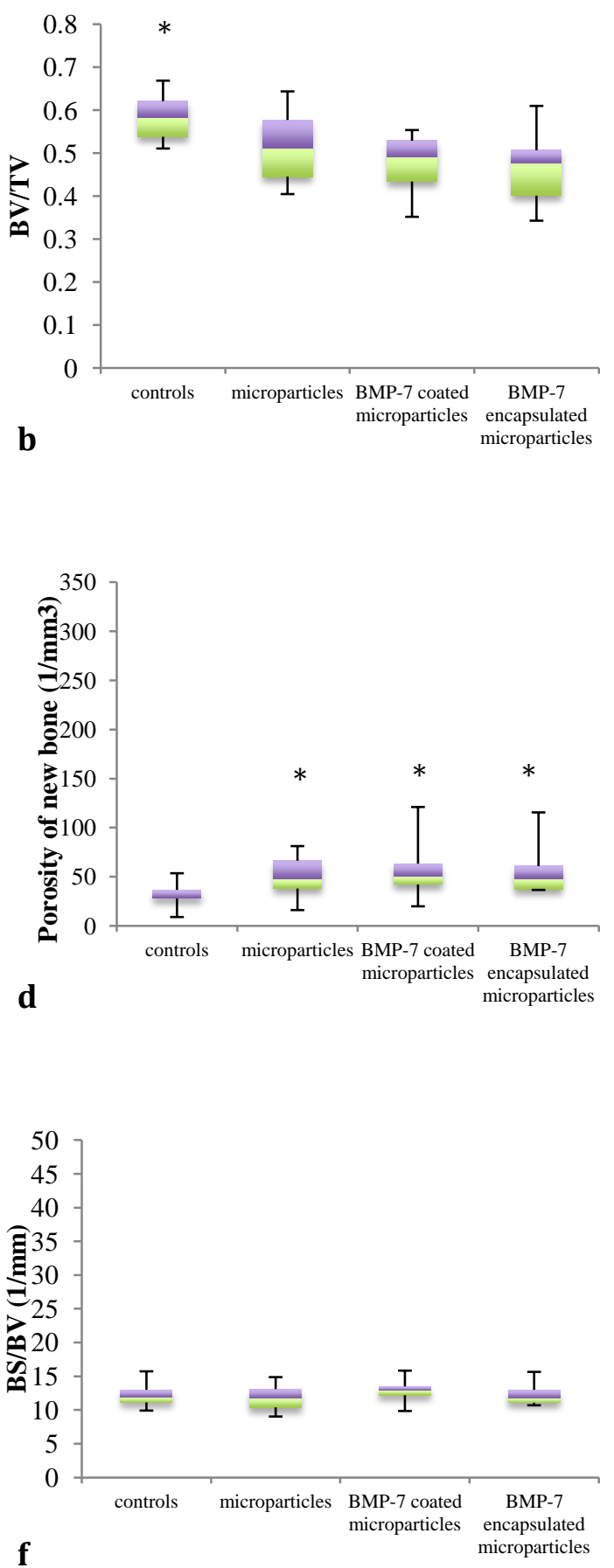
6 weeks
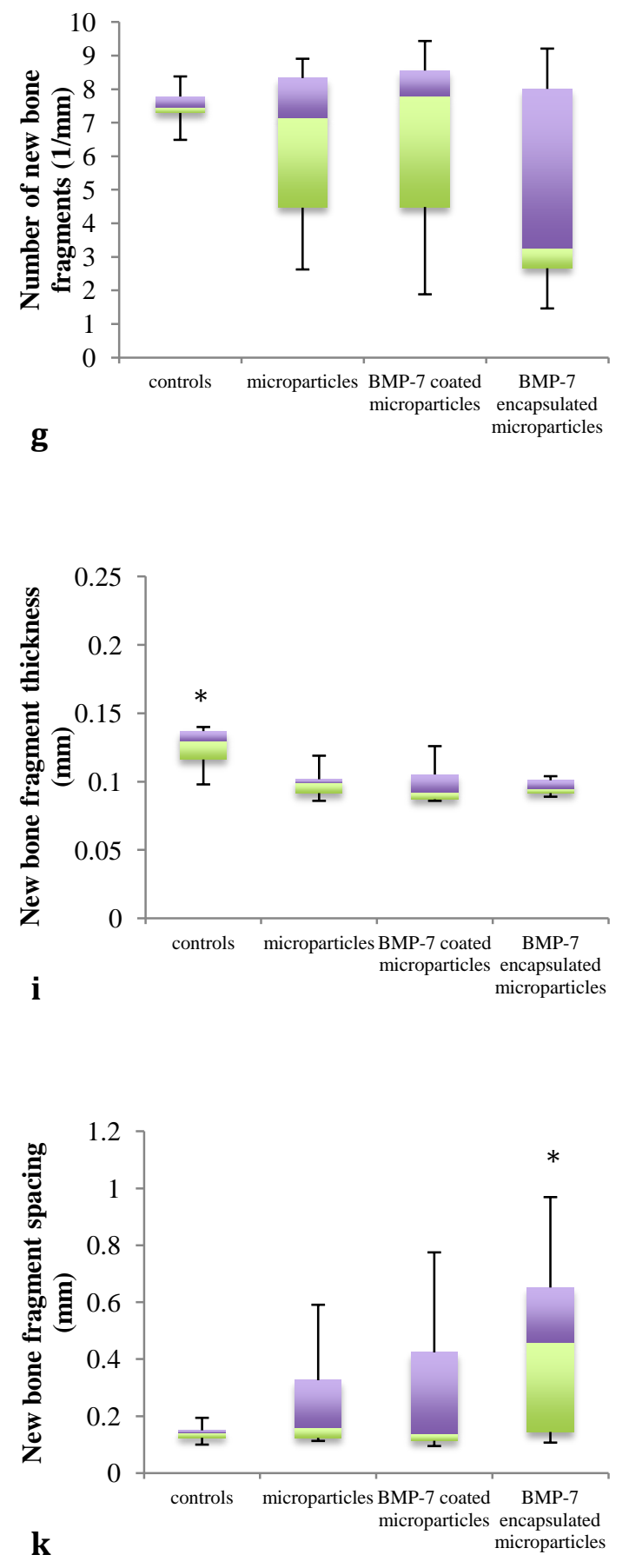

\section{2 weeks}
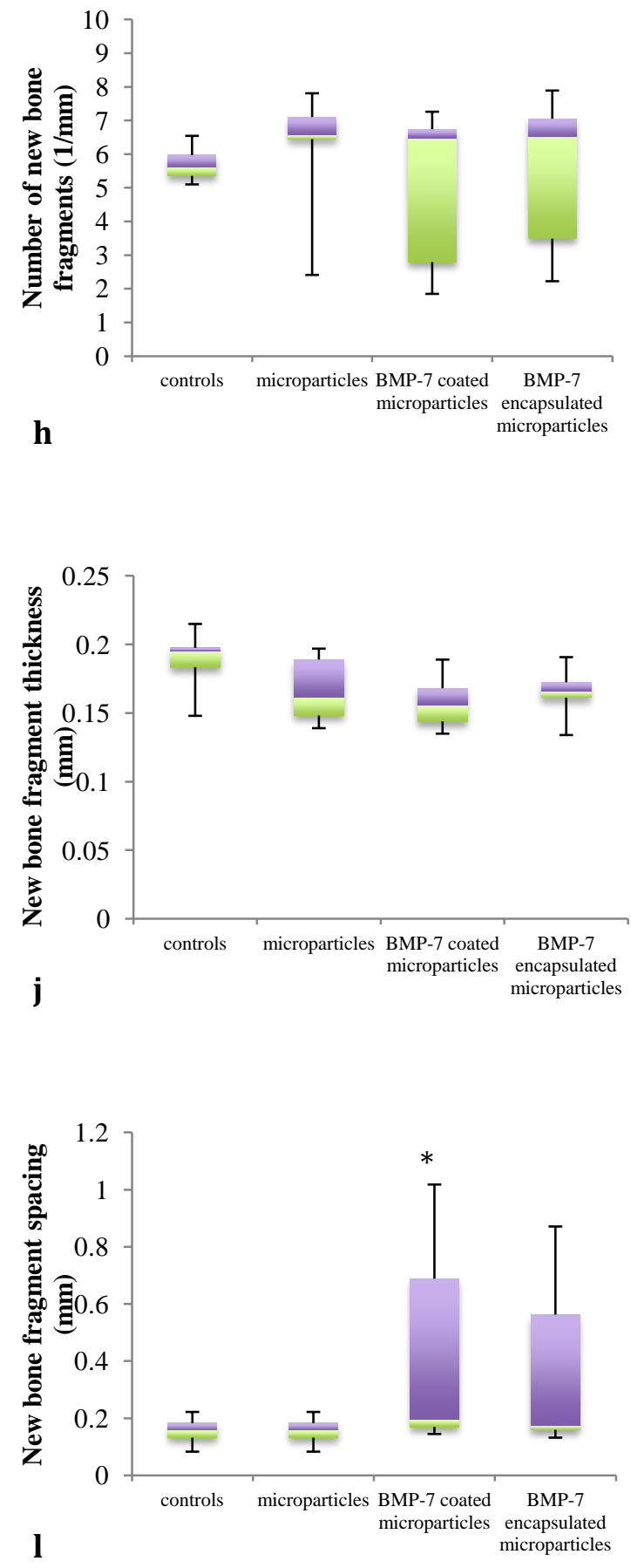

Fig.4 

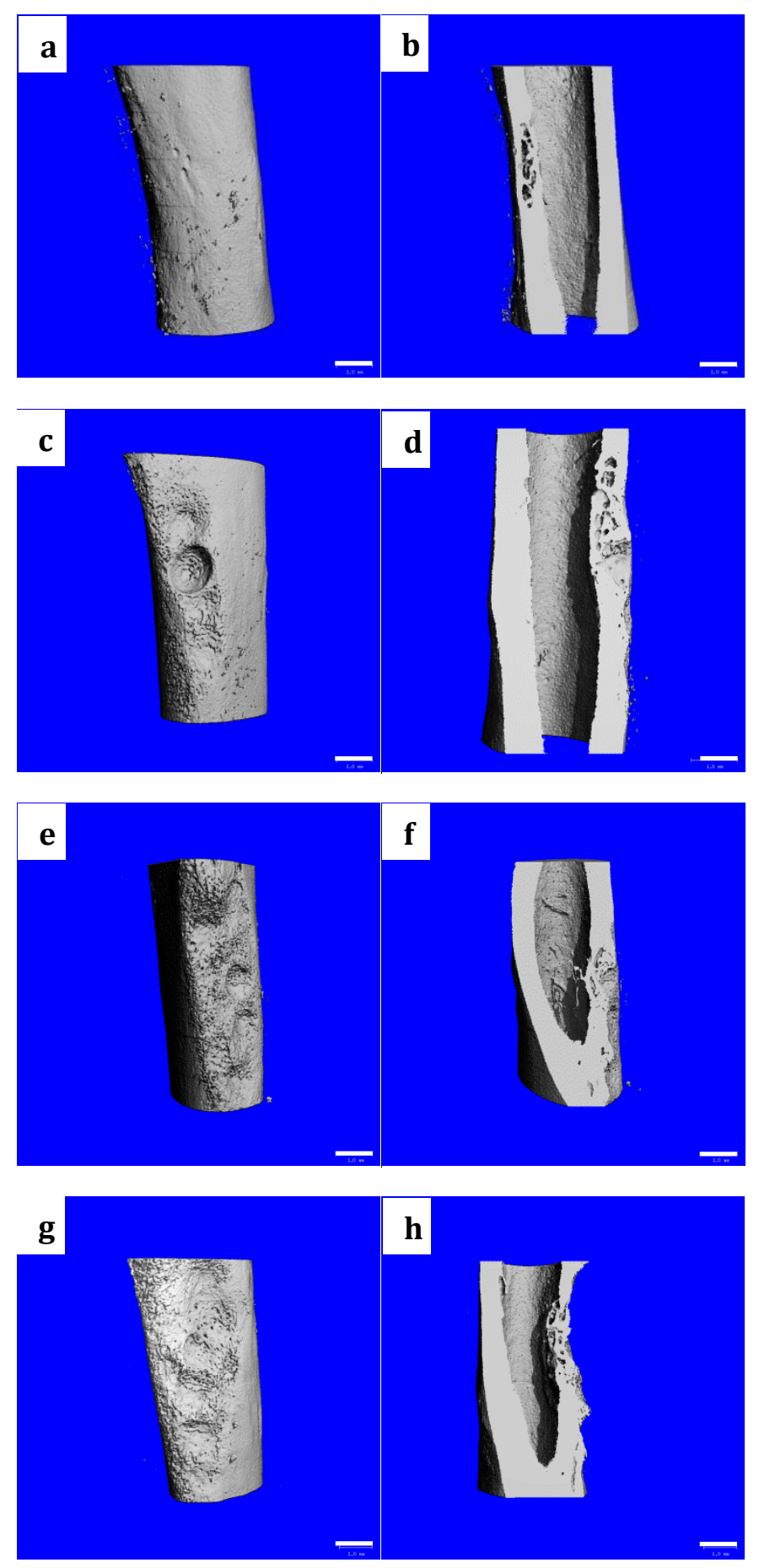

Fig.5 

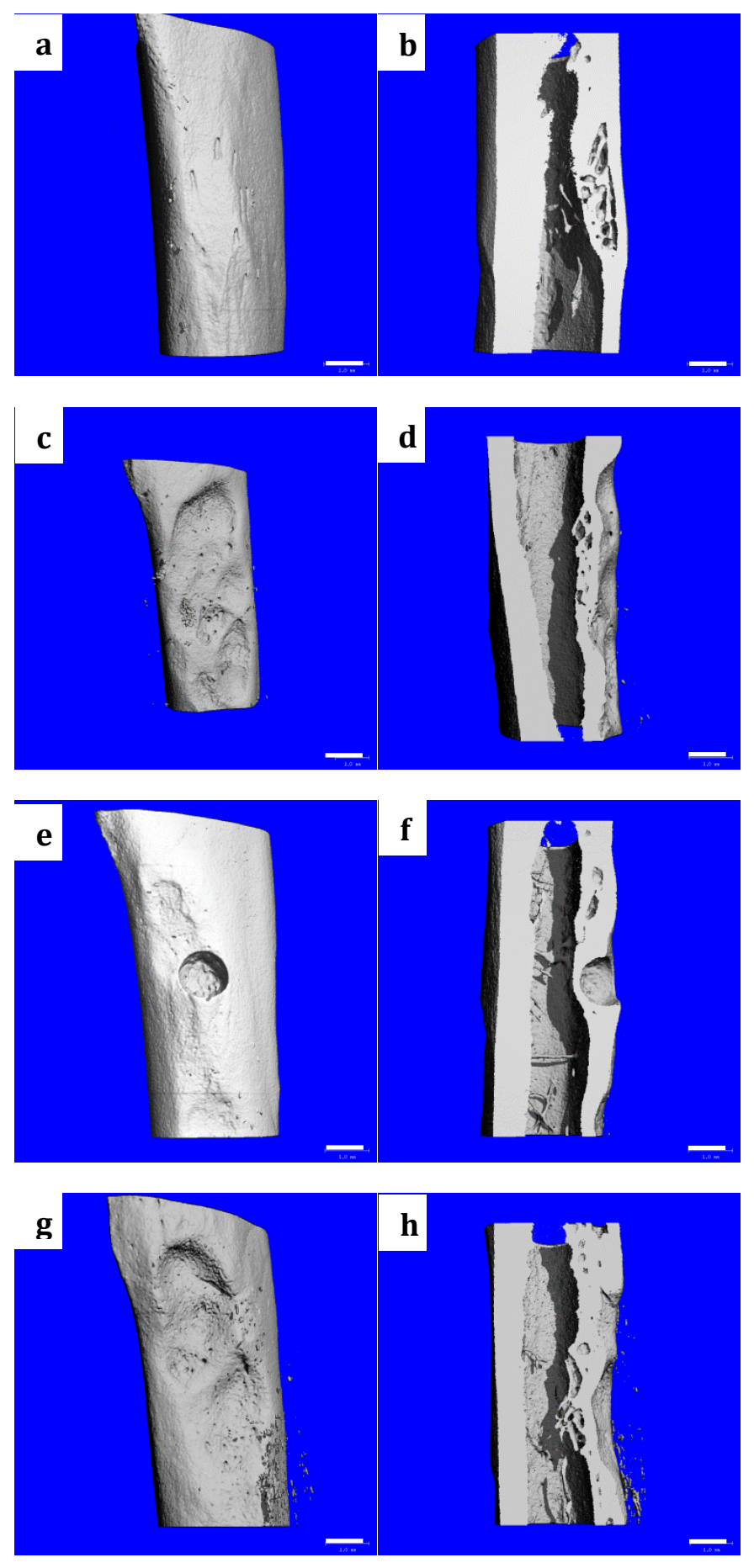

Fig.6 


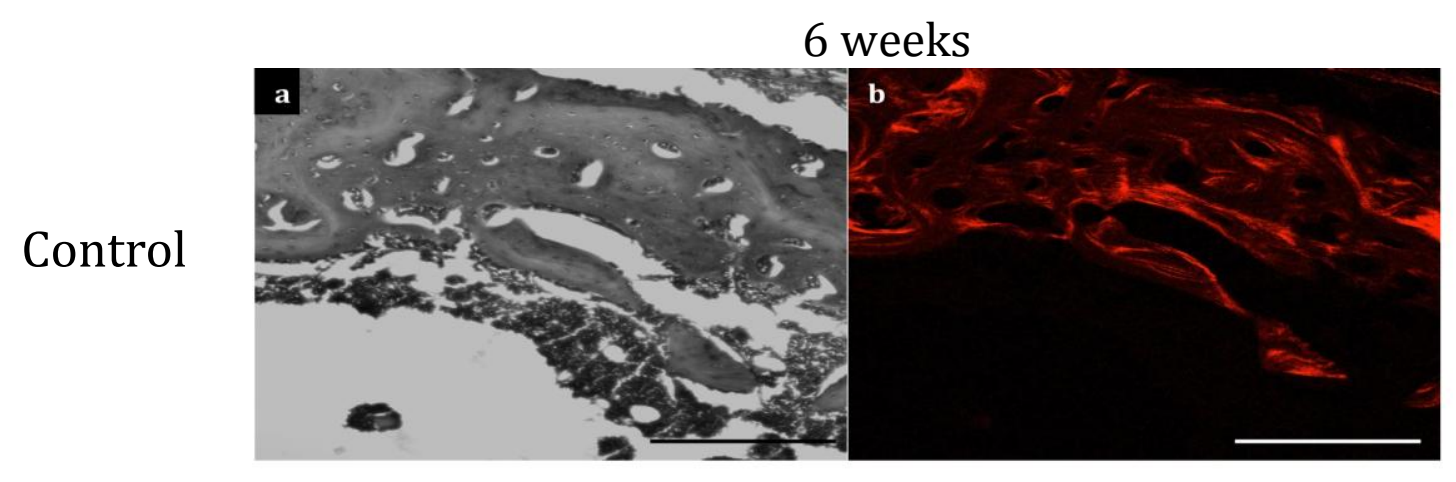

Microparticle
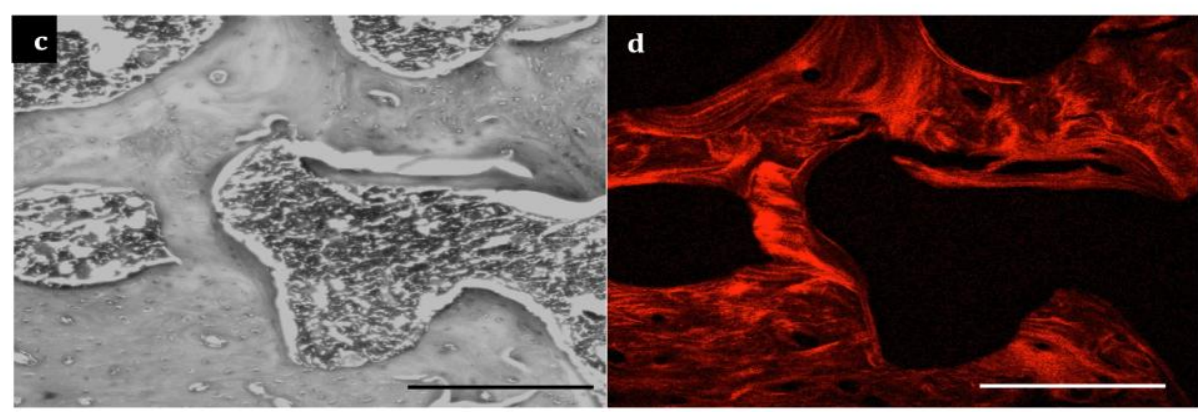

BMP-7 coated microparticles
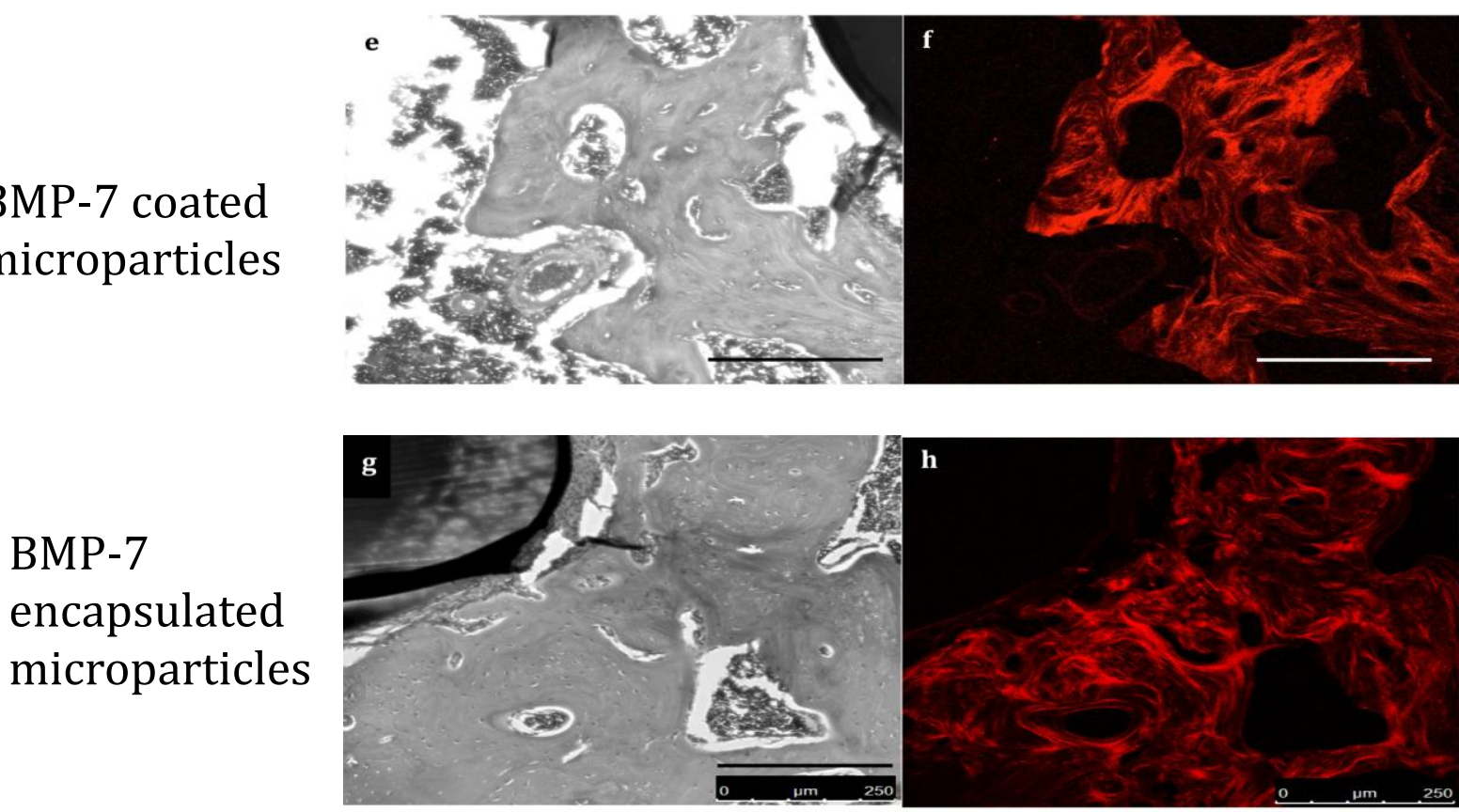

h

Fig.7 
12 weeks

Contro

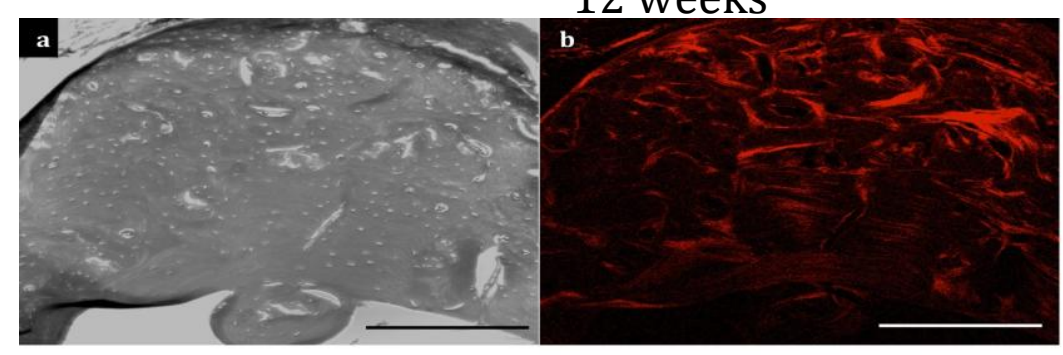

Microparticles
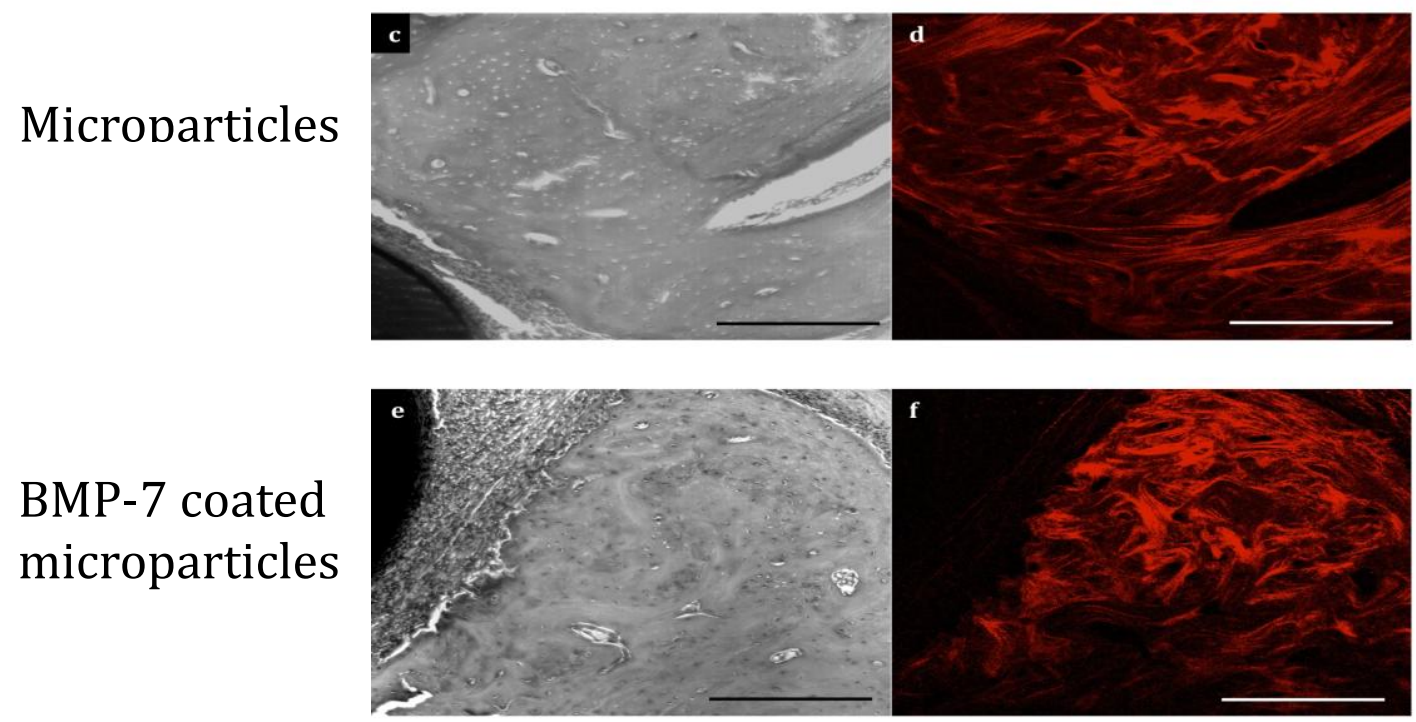

BMP-7

encapsulated microparticles

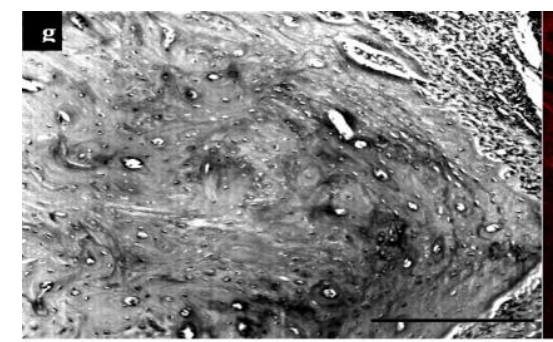



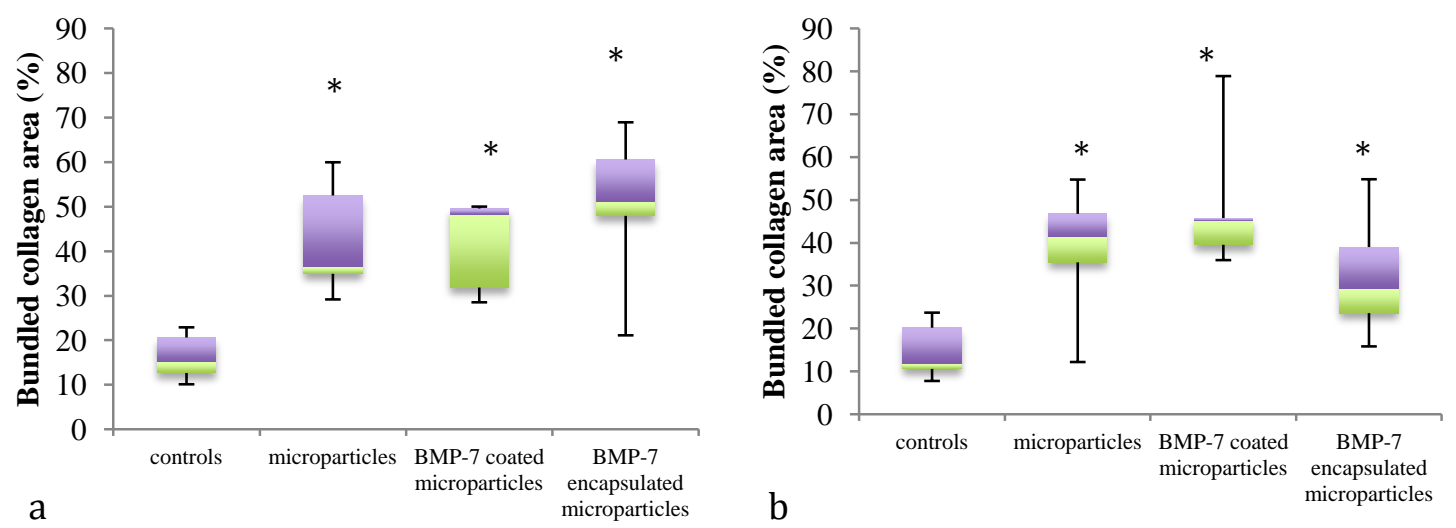

Fig. 9. 
Groups tested ( $n=10)$ : Chitosan microparticles

- Without growth factor

- BMP-7 coated

- BMP-7 encapsulated

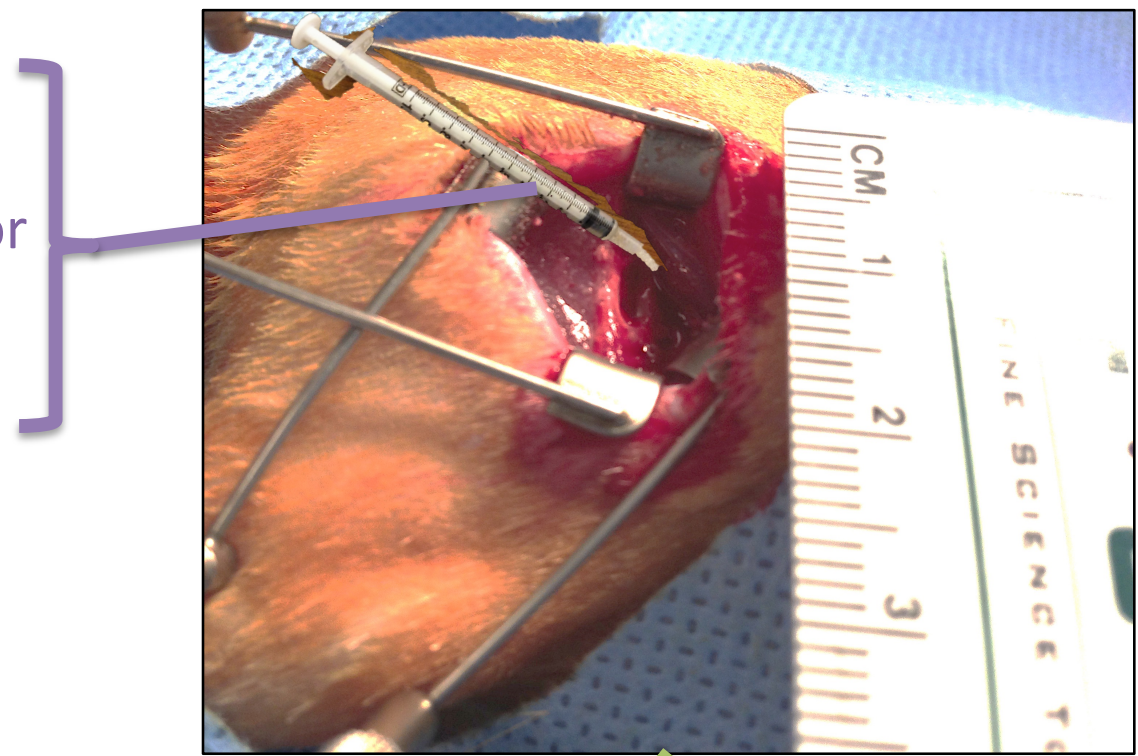

Rat femoral defect model

@ 6 \& 12 weeks, regenerated bone analyzed

\section{Qualitative analysis Quantitative analysis}

\section{Histology}
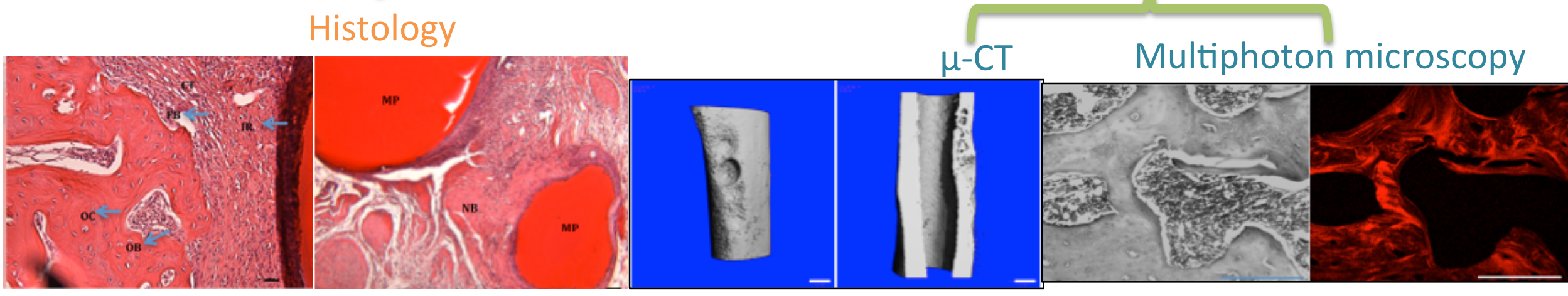